\title{
Rational approximation on quadrics: a simplex lemma and its consequences
}

\author{
Dmitry Kleinbock* and Nicolas de Saxcé
}

\begin{abstract}
We give elementary proof of stronger versions of several recent results on intrinsic Diophantine approximation on rational quadric hypersurfaces $X \subset \mathbb{P}^{n}(\mathbb{R})$. The main tool is a refinement of the simplex lemma, which essentially says that rational points on $X$ which are sufficiently close to each other must lie on a totally isotropic rational subspace of $X$.
\end{abstract}

\section{Introduction}

The classical theory of Diophantine approximation studies the way points $\mathbf{x} \in$ $\mathbb{R}^{n}$ are approximated by rational points $\frac{\mathbf{p}}{q} \in \mathbb{Q}^{n}$, taking into account the tradeoff between the size of $q$ and the distance between $\frac{\mathbf{p}}{q}$ and $\mathbf{x}$; see [6, 25] for a general introduction. Sometimes $\mathbf{x}$ is assumed to lie on a certain subset of $\mathbb{R}^{n}$, for example a smooth manifold $X$; this leads to the theory of Diophantine approximation on manifolds, in which there is no distinction between rational points which do or do not lie in $X$ (this is referred to as ambient approximation).

Let now $X$ be a rational quadric hypersurface of $\mathbb{R}^{n}$, let $\mathrm{x} \in X$ and let $\frac{\mathbf{p}}{q} \in \mathbb{Q}^{n}$ be such that the distance between $\mathbf{x}$ and $\frac{\mathbf{p}}{q}$ is less than $\psi(q)$, where $\psi$ is decaying fast enough, namely $\lim _{x \rightarrow \infty} x^{2} \psi(x)=0$. Then $\frac{\mathbf{p}}{q}$ must lie on $X$ whenever $q$ is large enough! This elementary observation, due to Dickinson and Dodson [11] for $n=2$ and more generally to Druţu, see [12, Lemma 4.1.1], has in part motivated a new field of intrinsic approximation, which examines the quality to which points on a manifold are approximated by rational points lying on that same manifold. The paper [20] studies the case $X=\mathbb{S}^{n-1}$, the unit sphere in $\mathbb{R}^{n}$. Later in [13] the results of [20] were significantly strengthened and extended to the case of $X$ being an arbitrary rational quadric hypersurface. An even more general framework was developed in 14]. Roughly speaking, in order to exhibit points on submanifolds $X \subset \mathbb{R}^{n}$ which are close enough to rational points of $X$, one has to make use of the structure of $X$ (indeed, in general it is not even guaranteed that $X \cap \mathbb{Q}^{n}$ is not empty). On the other hand, it is shown in 14 that to prove some negative results, that is, to show that many points of $X$ are not too close to rational points, one often does not need to know much about $X$. The main tool on which the argument of [14] is based is the Simplex Lemma originating in Davenport's work [10]. The version presented in [14, Lemma 4.1] is very general - it applies to any manifold embedded in $\mathbb{R}^{n}-$

\footnotetext{
${ }^{*}$ D.K. was supported in part by NSF grant DMS-1600814.
} 
and at the same time precise enough to yield some satisfying theorems in the case of quadric hypersurfaces.

The purpose of this note is to show that in the special case where $X$ is a rational quadric hypersurface, one can give more elementary and more geometric proofs of the results of [14]. This new approach will also yield more precise theorems. The main point is that one can prove a version of the simplex lemma with arbitrary hyperplanes replaced by $\mathbb{Q}$-isotropic subspaces of $X$; this, in turn, yields refined information on the diophantine properties of $X$.

A detailed account of the results that are derived here is given in the next section. After that in 3 we prove the simplex lemma for quadrics, Lemma 3.1 , which is central in all the subsequent developments. Applications of the simplex lemma to Diophantine approximation on quadrics are presented in $\$ 4$. Those results are proved along the same lines as the analogous statements for Diophantine approximation in the Euclidean space $\mathbb{R}^{n}$, but the proofs are included to make the paper self-contained. Finally, in $\$ 5$ we discuss some open problems and possible further directions for the study of intrinsic Diophantine approximation on projective varieties.

Acknowledgements. The authors are grateful to Emmanuel Breuillard, Nikolay Moshchevitin and Barak Weiss for motivating discussions and suggestions.

\section{General setting and main results of the paper}

Since it will make the proofs more transparent, we shall from now on always work in the projective setting. We denote by $\mathbb{P}^{n}(\mathbb{R})$ the $n$-dimensional real projective space. The natural map from $\mathbb{R}^{n+1}$ to $\mathbb{P}^{n}(\mathbb{R})$ will be denoted by $\mathbf{x} \mapsto[\mathbf{x}]$. For $x, y$ in $\mathbb{P}^{n}(\mathbb{R})$ the distance between $x$ and $y$ is given by

$$
\operatorname{dist}(x, y):=\frac{\left\|\mathbf{v}_{x} \wedge \mathbf{v}_{y}\right\|}{\left\|\mathbf{v}_{x}\right\|\left\|\mathbf{v}_{y}\right\|}
$$

where $\mathbf{v}_{x}$ and $\mathbf{v}_{y}$ are any nonzero vectors on $x$ and $y$, respectively, and $\|\cdot\|$ is the Euclidean norm. If $v=[\mathbf{v}] \in \mathbb{P}^{n}(\mathbb{Q})$, where $\mathbf{v}=\left(v_{1}, \ldots, v_{n+1}\right)$ is an integer vector with coprime coordinates, the height of $v$ is simply

$$
H(v):=\max _{1 \leq i \leq n+1}\left|v_{i}\right| .
$$

Given a point $x$ in $\mathbb{P}^{n}(\mathbb{R})$ we want to study how well $x$ is approximated by points $v$ in $\mathbb{P}^{n}(\mathbb{Q})$.

Remark 2.1. In order to go back to the setting of $\mathbb{R}^{n}$, one can consider an affine chart from an open subset of $\mathbb{P}^{n}(\mathbb{R})$ to $\mathbb{R}^{n+1}$. For example, if

$$
U=\left\{\left[\left(x_{1}, \ldots, x_{n+1}\right)\right]: x_{n+1} \neq 0\right\},
$$

one can use the chart

$$
\begin{array}{ccc}
U & \stackrel{\Phi}{\longmapsto} & \mathbb{R}^{n}, \\
{\left[\left(x_{1}, \ldots, x_{n+1}\right)\right] \stackrel{\Phi}{\longmapsto}} & \left(\frac{x_{1}}{x_{n+1}}, \ldots, \frac{x_{n}}{x_{n+1}}\right) .
\end{array}
$$


We consider a projective rational quadric $X$, given as the set of zeros of a rational quadratic form $Q$ in $n+1$ variables. Namely, for such $Q$ let us consider

$$
X=\left[Q^{-1}(0)\right]=\left\{x \in \mathbb{P}^{n}(\mathbb{R}): x=[\mathbf{x}] \text { with } Q(\mathbf{x})=0\right\} .
$$

Let us say that a subspace $E \subset \mathbb{R}^{n+1}$ is totally isotropic (with respect to $Q)$ if $\left.Q\right|_{E} \equiv 0$. If $E$ is as above, the projection $[E] \subset X$ of $E$ onto $\mathbb{P}^{n}(\mathbb{R})$ will be referred to as a totally isotropic projective subspace. Recall that the $\mathbb{Q}$-rank $\operatorname{rk}_{\mathbb{Q}} X$ of the quadric $X$ is the maximal dimension of a totally isotropic rational subspace of $\mathbb{R}^{n+1}$. If $\operatorname{rk}_{\mathbb{Q}} X>0$, this is the same as the maximal dimension of a totally isotropic rational projective subspace of $X$ plus one. In particular, $\operatorname{rk}_{\mathbb{Q}} X>0$ if and only if $X(\mathbb{Q}) \neq \varnothing$.

Example 2.2. As an example of a transition between affine and projective quadrics, let us consider the case $X=\mathbb{S}^{n-1}$. The affine sphere

$$
Y=\left\{\left(y_{1}, \ldots, y_{n}\right): y_{1}^{2}+\cdots+y_{n}^{2}=1\right\}
$$

can be identified with

$$
X=\left\{\left[\left(x_{1}, \ldots, x_{n+1}\right)\right]: x_{1}^{2}+\cdots+x_{n}^{2}=x_{n+1}^{2}\right\}
$$

via the map $\Phi$ described in Remark 2.1. Here $Q(\mathbf{x})=x_{1}^{2}+\cdots+x_{n}^{2}-x_{n+1}^{2}$, and the $\mathbb{Q}$-rank of $X$ is equal to 1 , with rational points of $X$ (or of $Y$ ) being in oneto-one correspondence with rational lines in $\mathbb{R}^{n+1}$ which are totally isotropic with respect to $Q$.

Given a point $x$ in $X$, we shall be interested in the quality of rational approximations $v \in X(\mathbb{Q})$ to $x$. Informally speaking, $v$ constitutes a reasonably good approximation to $x$ if $\operatorname{dist}(x, v)$ is small and the height of $v$ is not too large. Note that if $v=[(\mathbf{p}, q)]$, where $(\mathbf{p}, q)$ is a vector in $\mathbb{Z}^{n+1}$ with coprime coordinates, and $\Phi$ is the map defined in Remark 2.1] then $\Phi(v)=\mathbf{p} / q$. When $x$ is close to $v, \mathbf{y}=\Phi(x)$ is close to $\mathbf{p} / q$, therefore the ratio of $H(v)$ and $|q|$ is bounded between two constants depending only on $x$. The same can be said about the ratio of $\operatorname{dist}(x, v)$ and $\|\mathbf{y}-\mathbf{p} / q\|$. Thus the quality of rational approximations $v \in X(\mathbb{Q})$ to $x \in X$ is up to a multiplicative constant the same as the quality of rational approximations $\mathbf{p} / q \in Y=\Phi(X)$ to $\mathbf{y}=\Phi(x)$.

The basic theory of such approximations has been developed in 13 by Fishman, Kleinbock, Merrill and Simmons. In particular it was proved there 13 , Theorem 5.1] that if

$$
\operatorname{rk}_{\mathbb{Q}} X>0 \text { and } X \text { is nonsingular }
$$

(recall that a quadric hypersurface $X$ is said to be nonsingular if the quadratic form that defines it is nondegenerate, i.e. has nonzero discriminant 1 ), then for every $x \in X$ there exists $C_{x}>0$ and a sequence $\left(v_{k}\right)_{1}^{\infty}$ in $X(\mathbb{Q})$ such that

$$
v_{k} \rightarrow x \quad \text { and } \quad \operatorname{dist}\left(v_{k}, x\right) \leq \frac{C_{x}}{H\left(v_{k}\right)}
$$

Previously this was established in [20] by Kleinbock and Merrill for $X=\mathbb{S}^{n-1}$, in which case the constant $C_{x}$ can be chosen independently of $x$. In other words,

\footnotetext{
${ }^{1}$ This is also equivalent to $X$ being nonsingular as a projective algebraic variety.
} 
in the affine language of Example 2.2 there exists a constant $C$ dependent only on $n$ such that for any $\mathbf{y} \in \mathbb{S}^{n-1}$ there exist infinitely many $\mathbf{p} / q \in \mathbb{S}^{n-1}$ with $\|\mathbf{y}-\mathbf{p} / q\| \leq C /|q|$.

Now for a point $x$ in a quadric hypersurface $X \subset \mathbb{P}^{n}(\mathbb{R})$, let us define the (intrinsic) Diophantine exponent of $x$ by

$$
\beta(x):=\inf \left\{\beta>0 \mid \exists c>0: \forall v \in X(\mathbb{Q}), \operatorname{dist}(x, v) \geq c H(v)^{-\beta}\right\} ;
$$

then it follows that under the assumption (2.3), $\beta(x) \geq 1$ for all $x \in X$. On the other hand, it is shown in [14, Theorem 1.5] that the opposite inequality $\beta(x) \leq 1$ is true for Lebesgue-almost every $x \in X$ in the generality when $X$ is not just a rational quadric but an arbitrary non-degenerate hypersurface. Moreover, the same is true if the Lebesgue measure is replaced by an absolutely decaying measure (see 4.1 for definitions and more detail).

This naturally leads to a question of exhibiting other measures $\mu$ on $X$ such that $\beta(x) \leq 1$ for $\mu$-almost all $x \in X$. This is reminiscent to the subject of Diophantine approximation on manifolds and fractals, which has been extensively developed during recent decades for ambient approximation in $\mathbb{R}^{n}$, see [3, 18, 19]. Measures satisfying the above property are usually called extremal. We shall also say that a submanifold $M \subset X$ is extremal if so is the Lebesgue measure on $M$ (by which we mean the restriction to $M$ of the $k$-dimensional Hausdorff measure where $k=\operatorname{dim} M$ ).

Our first theorem, which is actually a special case of a more general result, Theorem 4.2. refines [14, Theorem 1.5] for rational quadrics $X$ as follows:

Theorem 2.3 (Extremality of submanifolds of large dimension). Let $X$ be $a$ rational quadric hypersurface in $\mathbb{P}^{n}(\mathbb{R})$, and let $M$ be a smooth submanifold of $X$ with $\operatorname{dim} M \geq \operatorname{rk}_{\mathbb{Q}} X$. Then $\beta(x) \leq 1$ for Lebesgue-almost every $x \in M$.

In the case where $X$ has $\mathbb{Q}$-rank one, the above theorem provides a very simple and satisfactory answer to the problem of Diophantine approximation on submanifolds of $X$ : any positive-dimensional submanifold $M \subset X$ is extremal. Note that there is no non-degeneracy condition on the submanifold $M$. This comes in contrast to the case of approximation in $\mathbb{R}^{n}$, where one has to require that the submanifold is not included in an affine subspace.

In view of Theorem 2.3, it is natural to ask, given a submanifold $M$ of $X$ of dimension at least $\operatorname{rk}_{\mathbb{Q}} X$ and a fixed $\beta>1$, how large the intersection $M \cap W_{\beta}$ can be, where $W_{\beta}$ denotes the set of points in $X$ whose Diophantine exponent is at least $\beta$. Note that it was proved in [13, Theorem 6.4] that whenever $X$ satisfies (2.3), the Hausdorff dimension of $W_{\beta}$ is equal to $\frac{n-1}{\beta}$. Also in [15] some upper estimates for the Hausdorff dimension of $M \cap W_{\beta}$ was obtained in the case when $M$ supports an absolutely decaying and Ahlfors-regular measure (see $\$ 4.2$ for details). Our second application of the simplex lemma strengthens the main result of [15]. Here is a special case of a more general result, Theorem 4.6:

Theorem 2.4 ( $\beta$-approximable points on submanifolds of large dimension). Let $X$ be a rational quadric hypersurface in $\mathbb{P}^{n}(\mathbb{R})$, and let $M$ be a $k$-dimensional smooth submanifold of $X$ with $k \geq \operatorname{rk}_{\mathbb{Q}} X$. Then one has

$$
\operatorname{dim}_{H}\left(M \cap W_{\beta}\right) \leq k-\left(k+1-\operatorname{rk}_{\mathbb{Q}} X\right)\left(1-\frac{1}{\beta}\right) .
$$


As the third application of our simplex lemma, we study the winning property of the set $\mathrm{BA}_{X}$ of badly approximable points on $X$. In our setting,

$$
\mathrm{BA}_{X}:=\left\{x \in X \mid \exists c>0: \forall v \in X(\mathbb{Q}), \operatorname{dist}(x, v) \geq c H(v)^{-1}\right\} .
$$

We define a version of Schmidt's game using only totally isotropic rational subspaces and show the associated winning property for the set $\mathrm{BA}_{X}$ (Theorem 4.10). Here is a special case:

Theorem 2.5 (Thickness of $\mathrm{BA}_{X}$ on submanifolds of large dimension). Let $X$ be a rational quadric hypersurface in $\mathbb{P}^{n}(\mathbb{R})$. Then for any $C^{1}$ submanifold $M \subset X$ of dimension at least $\operatorname{rk}_{\mathbb{Q}} X$,

$$
\operatorname{dim}_{H}\left(\mathrm{BA}_{X} \cap M\right)=\operatorname{dim} M .
$$

The properties of the set $\mathrm{BA}_{X}$ have been studied in [14. In particular, it was shown [14, Theorem 4.3] that $\mathrm{BA}_{X}$ is hyperplane absolute winning (see 4.3 for the definition and more detail); this gave the conclusion of the above theorem for $M=X$. The refined version given above has the advantage that it is optimal: indeed, if $M$ is any totally isotropic rational projective subspace of $X$ of dimension $\operatorname{rk}_{\mathbb{Q}} X-1$, then $\mathrm{BA}_{X} \cap M=\varnothing$.

\section{Diagonal flows and the simplex lemma}

The purpose of this section is to derive a simplex lemma, Lemma 3.1, for rational points on a rational quadric hypersurface $X \subset \mathbb{R}^{n+1}$. For the proof, we shall relate good rational approximations to $x \in X$ to the behavior of some diagonal orbit in the space of lattices in $\mathbb{R}^{n+1}$.

Recall that the classical simplex lemma 2 states that for each $n \in \mathbb{N}$ there exists $c=c(n)>0$ such that if $x$ is a point in $\mathbb{P}^{n}(\mathbb{R})$ and $\rho \in(0,1)$, then there exists an $(n-1)$-dimensional projective subspace of $\mathbb{P}^{n}(\mathbb{R})$ containing all rational points with height at most $c \rho^{-\frac{n}{n+1}}$ inside the ball $B(x, \rho)$. For a proof of the simplex lemma, we refer the reader to [22, Lemma 4].

Here we consider a rational quadratic form $Q$ on $\mathbb{R}^{d}$ and study rational points on $X$ as in (2.1).

Lemma 3.1 (Simplex lemma for quadric hypersurfaces). Let $X$ be a rational quadric hypersurface in $\mathbb{P}^{n}(\mathbb{R})$. Then there exists $c>0$ such that, for every ball $B_{\rho} \subset X$ of radius $\rho \in(0,1)$, the set

$$
B_{\rho} \cap\left\{v \in X(\mathbb{Q}) \mid H(v) \leq c \rho^{-1}\right\}
$$

is contained in a totally isotropic rational projective subspace of $X$.

Let $B_{Q}$ be the symmetric bilinear form associated to the quadratic form $Q$ defining $X$. The kernel of $Q$ is defined by

$$
\operatorname{ker} Q=\left\{x=[\mathbf{x}] \in \mathbb{P}^{n}(\mathbb{R}) \mid \forall \mathbf{y} \in \mathbb{R}^{n+1}, B_{Q}(\mathbf{x}, \mathbf{y})=0\right\} \text {. }
$$

Assuming that $X(\mathbb{Q}) \backslash \operatorname{ker} Q$ is non-empty, we may write, in some rational basis of $\mathbb{R}^{n+1}$,

$$
Q\left(x_{1}, \ldots, x_{n+1}\right)=2 x_{1} x_{n+1}+\tilde{Q}\left(x_{2}, \ldots, x_{n}\right),
$$

\footnotetext{
${ }^{2}$ Here we restate it using the projective language.
} 
where $\tilde{Q}$ is a quadratic form in $n-1$ variables. Let $G=\mathrm{SO}_{Q}(\mathbb{R})$ be the group of unimodular linear transformations of $\mathbb{R}^{n+1}$ preserving the quadratic form $Q$. The group $G$ acts transitively on $X \backslash \operatorname{ker} Q$, which may be identified with the quotient space $X \simeq P \backslash G$, where $P$ is the stabilizer of the isotropic line [ $\mathbf{e}_{1}$ ] in the standard representation. In fact, for $x \in X \backslash \operatorname{ker} Q$, we may choose $u_{x} \in G \cap O_{n+1}(\mathbb{R})$ such that $u_{x} x=\left[\mathbf{e}_{1}\right]$.

We shall consider the diagonal subgroup $a_{t}=\operatorname{diag}\left(e^{-t}, 1, \ldots, 1, e^{t}\right)$ in $G$, and if $x \in X$, let

$$
g_{t}^{x}=u_{x}^{-1} a_{t} u_{x} .
$$

The lemma below is due to Kleinbock and Merrill [20] in the case of projective spheres, and to Fishman, Kleinbock, Merrill and Simmons [13, Lemma 7.1] in the general case.

Lemma 3.2 (Dani correspondence for quadric hypersurfaces). Let $Q$ be as in (3.1), and write $X$ for the associated rational quadric hypersurface in $\mathbb{P}^{n}(\mathbb{R})$. With the above notation, there exists $C>0$ such that for $x \in X$ and $v \in X$, we have, for all $t \in \mathbb{R}$,

$$
\left\|g_{t}^{x} \mathbf{v}\right\| \leq C \max \left(e^{-t} H(v), H(v) \operatorname{dist}(x, v), e^{t} H(v) \operatorname{dist}(x, v)^{2}\right),
$$

where $\mathbf{v} \in \mathbb{Z}^{d}$ is a representant of $v$ with coprime integer coordinates.

Proof. Since $\tilde{Q}$ is a quadratic form, we may choose $C_{0} \geq 2$ such that for all $\mathbf{w}$ in $\mathbb{R}^{n-1},|\tilde{Q}(\mathbf{w})| \leq C_{0}\|\mathbf{w}\|^{2}$. With $u_{x}$ as above, write

$$
u_{x} \mathbf{v}=v_{1} \mathbf{e}_{1}+v_{2} \mathbf{e}_{2}+\cdots+v_{n+1} \mathbf{e}_{n+1} .
$$

Letting $\mathbf{w}=v_{2} \mathbf{e}_{2}+\cdots+v_{n} \mathbf{e}_{n}$, we have

$$
u_{x} g_{t}^{x} \mathbf{v}=e^{-t} v_{1} \mathbf{e}_{1}+\mathbf{w}+e^{t} v_{n+1} \mathbf{e}_{n+1},
$$

and therefore, since $u_{x}$ is in $O_{n+1}(\mathbb{R})$,

$$
\left\|g_{t}^{x} \mathbf{v}\right\| \leq 3 \max \left(e^{-t}\left|v_{1}\right|,\|\mathbf{w}\|, e^{t}\left|v_{n+1}\right|\right) .
$$

Of course, $\left|v_{1}\right| \leq H(v)$ and $\|\mathbf{w}\| \leq\left\|\mathbf{w}+v_{n+1} \mathbf{e}_{n+1}\right\| \leq H(v) \operatorname{dist}(x, v)$. Moreover, $Q\left(u_{x} \mathbf{v}\right)=0$ yields

$$
\left|v_{n+1}\right|=\frac{|\tilde{Q}(\mathbf{w})|}{2\left|v_{1}\right|} \leq \frac{C_{0}\|\mathbf{w}\|^{2}}{2\left|v_{1}\right|}
$$

so that, provided $\operatorname{dist}(x, v) \leq \frac{\sqrt{2}}{2}$,

$$
\left|v_{d}\right| \leq \frac{C_{0}}{2} \frac{H(v) \operatorname{dist}(x, v)^{2}}{\sqrt{1-\frac{\operatorname{dist}(x, v)^{2}}{H(v)^{2}}}} \leq C_{0} H(v) \operatorname{dist}(x, v)^{2} .
$$

Of course, if $\operatorname{dist}(x, v) \geq \frac{\sqrt{2}}{2}$, we also have $\left|v_{d}\right| \leq H(v) \leq C_{0} H(v) \operatorname{dist}(x, v)^{2}$, because $C_{0} \geq 2$. Going back to (3.2), we find the desired inequality, with $C=3 C_{0}$.

We can now prove the simplex lemma. 
Proof of Lemma 3.1. Let $Q$ be a quadratic form defining the hypersurface $X$. The result is obvious if $X(\mathbb{Q}) \subset \operatorname{ker} Q$, so we may assume that $X(\mathbb{Q}) \backslash \operatorname{ker} Q$ is non-empty. Then, replacing $Q$ if necessary by an integer multiple, we may find an integer basis of $\mathbb{R}^{n+1}$ in which $Q$ has the form (3.1).

Fix a constant $C_{1}$ such that for all $\mathbf{v} \in \mathbb{R}^{n+1},|Q(\mathbf{v})| \leq C_{1}\|\mathbf{v}\|^{2}$, and let $c=\frac{1}{C \sqrt{5 C_{1}}}$, where $C$ is the constant given by Lemma 3.2. We need to show that any family $v_{1}, \ldots, v_{s}$ of points in $X(\mathbb{Q}) \cap B(x, \rho)$ satisfying $H\left(v_{i}\right) \leq c \rho^{-1}$, $i=1, \ldots, s$, generates a totally isotropic subspace. For each $v_{i}$, we take a representant $\mathbf{v}_{i}$ in $\mathbb{Z}^{n+1}$ with coprime integer coordinates. It is enough to show that for all $i$ and $j, Q\left(\mathbf{v}_{i} \pm \mathbf{v}_{j}\right)=0$, and since the quadratic form $Q$ takes integer values at integer points, it suffices to check that for all $i$ and $j,\left|Q\left(\mathbf{v}_{i} \pm \mathbf{v}_{j}\right)\right|$ is less than 1.

Now, choosing $t>0$ such that $e^{t}=\rho^{-1}$, Lemma3.2 shows that $\left\|g_{t}^{x} \mathbf{v}_{i}\right\| \leq C c$. Then, we write

$$
\left.Q\left(\mathbf{v}_{i} \pm \mathbf{v}_{j}\right)=Q\left(g_{t}^{x} \mathbf{v}_{i} \pm g_{t}^{x} \mathbf{v}_{j}\right)\right) \leq C_{1}\left\|g_{t}^{x} \mathbf{v}_{i} \pm g_{t}^{x} \mathbf{v}_{j}\right\|^{2} \leq 4 C_{1}(C c)^{2}=\frac{4}{5} .
$$

This implies what we want.

Remark 3.3. In the case when $X=\mathbb{S}^{n-1}$ is the $(n-1)$-dimensional sphere, identified with $Y$ as in (2.2), one can give a more direct proof of the simplex lemma. Indeed, if $\frac{\mathbf{p}_{1}}{q_{1}}$ and $\frac{\mathbf{p}_{2}}{q_{2}}$ are two distinct rational points on $\mathbb{S}^{n-1}$ of height at most $\frac{\rho^{-1}}{2}$, we have

$$
\left\|\frac{\mathbf{p}_{1}}{q_{1}}-\frac{\mathbf{p}_{2}}{q_{2}}\right\|^{2}=2-\frac{\left(\mathbf{p}_{1}, \mathbf{p}_{2}\right)}{q_{1} q_{2}} \geq \frac{1}{q_{1} q_{2}} \geq 4 \rho^{2},
$$

so that any open ball of radius $\rho$ contains at most one rational point of height at most $\frac{\rho^{-1}}{2}$. In fact, such a direct computation can also be made for a general quadric hypersurface, but we chose to give a more geometric proof of Lemma 3.1 here.

Remark 3.4. When the quadratic form $Q$ has $\mathbb{Q}$-rank one, the only isotropic rational projective subspaces are points in $X(\mathbb{Q})$. This makes the consequences of the simplex lemma more spectacular in the particular case of $\mathbb{Q}$-rank one.

\section{Applications to Diophantine approximation}

In this section, as before, $X$ is a rational quadric hypersurface in $\mathbb{P}^{n}(\mathbb{R})$ defined by a rational quadratic form $Q$. We are concerned with intrinsic Diophantine approximation on $X$, which is the study of the quality of approximations of a point $x$ in $X$ by rational points $v$ lying on $X$. On that matter, the simplex lemma has several simple consequences, which we now explain.

\subsection{Extremality}

Recall that the Diophantine exponent of a point $x \in X$ was defined by (2.5). Our next theorem generalizes Theorem 2.3 using the following definition. 
Definition 4.1. Given a positive parameter $\alpha$, a finite Borel measure $\mu$ on the quadric hypersurface $X$ will be called $\alpha$-isotropically absolutely decaying, abbreviated as $\alpha-I A D$, if there exists a constant $C>0$ such that for every $x \in X$ and every totally isotropic rational projective subspace $L \subset X$,

$$
\forall \varepsilon>0 \forall \rho \in(0,1), \quad \mu\left(B(x, \rho) \cap L^{(\varepsilon \rho)}\right) \leq C \varepsilon^{\alpha} \mu(B(x, \rho)),
$$

where $L^{(\tau)}$ denotes the neighborhood of size $\tau$ of the set $L$. We shall say that $\mu$ is isotropically absolutely decaying (IAD) if it is $\alpha$-IAD for some $\alpha>0$.

Theorem 4.2 (IAD measures are extremal). Let $X$ be a rational quadric in $\mathbb{P}^{n}(\mathbb{R})$, and let $\mu$ be an IAD measure on $X$. Then $\beta(x) \leq 1$ for $\mu$-almost every $x \in X$.

Remark 4.3. Recall that a measure $\mu$ is called $\alpha$-absolutely decaying if (4.1) holds for some $C>0$, every $x \in X$ and every subspace $L \subset \mathbb{P}^{n}(\mathbb{R})$, and absolutely decaying if it is $\alpha$-absolutely decaying for some $\alpha>0$. It follows from [14. Theorem 1.5] that for any absolutely decaying measure $\mu$ on $X$ one has $\beta(x) \leq 1$ for $\mu$-almost every $x \in X$. In fact it holds more generally when $X$ is not just a rational quadric but an arbitrary non-degenerate smooth hypersurface.

Clearly absolutely decaying measures are IAD but not vice versa. In particular, it is clear that Lebesgue measure on a smooth proper submanifold $M$ of $X$ with $\operatorname{dim} M \geq \operatorname{rk}_{\mathbb{Q}} X$ is not absolutely decaying but $\alpha$-IAD with $\alpha=\operatorname{dim} M-\operatorname{rk}_{\mathbb{Q}} X+1$; so Theorem 2.3 is a corollary from Theorem 4.2 .

Proof of Theorem 4.2. The argument follows the lines of the proof of 23, Theorem 1], see also [26] for a one-dimensional version. By the Borel-Cantelli lemma, it is enough to check that for all $\varepsilon>0$,

$$
\sum_{k \geq 1} \mu\left(\left\{x \in X \mid \exists v \in X(\mathbb{Q}):\left\{\begin{array}{c}
2^{k} \leq H(v)<2^{k+1} \\
\operatorname{dist}(x, v) \leq 2^{-k(1+\varepsilon)}
\end{array}\right\}\right)<\infty .\right.
$$

Fix $k \geq 1$. There exists an integer $K$ such that we may cover $X$ by a family of balls $B_{i}=B\left(x_{i}, 2^{-k\left(1+\frac{\varepsilon}{2}\right)}\right), i=1, \ldots, N$, so that any intersection of more than $K$ distinct balls is empty. By Lemma 3.1, for $k$ large enough, for each $i$, the set of points $v \in X(\mathbb{Q}) \cap B_{i}$ satisfying $2^{k} \leq H(v)<2^{k+1}$ is contained in a totally isotropic rational subspace $L_{i}$, and therefore, by the IAD property of $\mu$ for some $C, \alpha>0$ one has

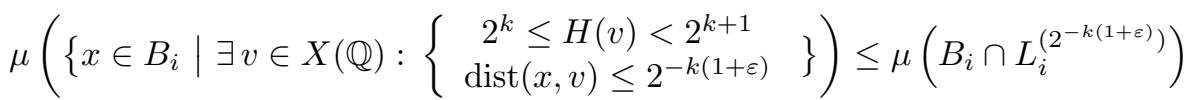

$$
\begin{aligned}
& \leq C 2^{-k \alpha \frac{\varepsilon}{2}} \mu\left(B_{i}\right) .
\end{aligned}
$$

Summing over all balls $B_{i}$, and using the fact that the cover $\left(B_{i}\right)_{i \in \mathbb{N}}$ has multiplicity at most $K$, we get

$$
\mu\left(\left\{x \in X \mid \exists v \in X(\mathbb{Q}):\left\{\begin{array}{c}
2^{k} \leq H(v)<2^{k+1} \\
\operatorname{dist}(x, v) \leq 2^{-k(1+\varepsilon)}
\end{array}\right\}\right) \leq K C 2^{-k \alpha \frac{\varepsilon}{2}} .\right.
$$

This finishes the proof of the theorem.

Remark 4.4. When $\operatorname{rk}_{\mathbb{Q}}(X)=1$, all the subspaces $L$ appearing in Definition 4.1 are zero-dimensional, and isotropic absolute decay coincides with weak absolute decay as defined in [2. Moreover, in the case where $X$ is a sphere, Theorem 4.2 can be viewed as a corollary of [2, Theorem 2]. 
Remark 4.5. We could have stated a slightly stronger version of the theorem, in the form of a Khintchine-type theorem: if $\mu$ is $\alpha$-IAD, and if $\psi: \mathbb{R}^{+} \rightarrow \mathbb{R}^{+}$ is a non-increasing function satisfying

$$
\sum_{k \in \mathbb{N}} k^{\alpha-1} \psi(k)^{\alpha}<\infty,
$$

then for $\mu$-almost every $x$ in $X$ there exists $c>0$ such that

$$
\forall v \in X(\mathbb{Q}), \operatorname{dist}(x, v) \geq c \psi(H(v)) .
$$

With some minor modifications, our proof works in this slightly more general setting.

\subsection{Hausdorff dimension and Diophantine exponents}

As a complement to the above study of the extremality problem, we explain here how the simplex lemma can be used to give a simple proof of a recent result of Fishman, Merrill and Simmons [15]. Once again, $X$ denotes a rational quadric projective hypersurface of dimension $n$. Given $\beta>0$, we shall be concerned with the set

$$
W_{\beta}=\{x \in X \mid \beta(x) \geq \beta\} .
$$

Given a subset $K$ in $X$, our goal will be to bound the Hausdorff dimension of the intersection $K \cap W_{\beta}$; we shall be able to do so if $K$ is the support of a sufficiently regular measure.

For $\delta>0$, a Borel measure $\mu$ on a metric space $X$ is said to be Ahlfors-regular of dimension $\delta$ if we have, for some constant $A>0$,

$$
\forall x \in X \forall r \in(0,1], \quad \frac{1}{A} r^{\delta} \leq \mu(B(x, r)) \leq A r^{\delta} .
$$

We now present a short proof of a strengthening of [15, Theorem 1.2], using Lemma 3.1 .

Theorem 4.6. Let $X$ be a rational quadric projective hypersurface. Let $\mu$ be an Ahlfors-regular measure of dimension $\delta$ on $X$, and let $K=\operatorname{Supp} \mu$. If $\mu$ is $\alpha-I A D$, then we have, for all $\beta \geq 1$,

$$
\operatorname{dim}_{H}\left(K \cap W_{\beta}\right) \leq \delta-\alpha\left(1-\frac{1}{\beta}\right) .
$$

Remark 4.7. 15, Theorem 1.2] establishes (4.2) under a stronger assumption that $\mu$ is $\alpha$-absolutely decaying. However in our decay condition we only have to consider totally isotropic subspaces. In particular, Theorem 4.6 covers the case where $K$ is a smooth submanifold of $X$ of dimension at least $\operatorname{rk}_{Q}(X)$, and therefore generalizes Theorem 2.4.

The proof of Theorem [4.6] is a straightforward adaptation of that of [23, Theorem 2]. We shall use the easy Hausdorff-Cantelli lemma stated below.

Lemma 4.8 (Hausdorff-Cantelli). Let $\left(B_{i}\right)_{i \geq 0}$ be a family of balls in a metric space, and assume that $\sum_{i \geq 0}\left(\operatorname{diam} B_{i}\right)^{s}<\infty$. Then,

$$
\operatorname{dim}_{H}\left(\lim \sup B_{i}\right) \leq s
$$


Proof. Left as an exercise, see Bernik-Dodson [3, Lemma 3.10].

Proof of Theorem 4.6. If $\beta=1$, there is nothing to prove, so we assume $\beta>1$ and fix $\gamma \in(1, \beta)$. For $p \geq 0$, let

$$
A_{p}=\left\{x \in X \mid \exists v \in X(\mathbb{Q}):\left\{\begin{array}{c}
2^{p} \leq H(v)<2^{p+1} \\
\operatorname{dist}(x, v) \leq 2^{-\gamma p}
\end{array}\right\} .\right.
$$

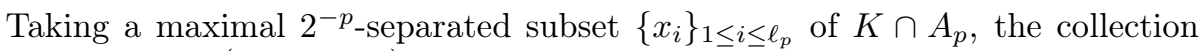
of balls $\mathcal{C}_{p}=\left(B\left(x_{i}, 2^{-p}\right)\right)_{1 \leq i \leq \ell_{p}}$ covers $K \cap A_{p}$ and has multiplicity bounded above by some constant $C$ depending only on $X$. Using the Ahlfors regularity of $\mu$, this implies $\ell_{p} 2^{-p \delta} \leq A C \mu(X)=A C$, i.e. $\ell_{p} \leq A C 2^{p \delta}$.

Since $\gamma>1$, Lemma 3.1 shows that for $p$ large enough, for each ball $B \in \mathcal{C}_{p}$, there exists a totally isotropic subspace $L_{B}$ of $X$ such that $A_{p} \cap B \subset L_{B}^{\left(2^{-\gamma p}\right)}$. So the decay condition on $\mu$ yields, within multiplicative constants depending only on $X$ and $\mu$, that

$$
\mu\left(A_{p} \cap B\right) \ll 2^{-(\gamma-1) \alpha p} \mu(B) \asymp 2^{-p[\delta+(\gamma-1) \alpha]} .
$$

Next, take a minimal cover $\mathcal{D}_{B}=\left(B_{i}\right)_{i \in I_{B}}$ of the set $K \cap A_{p} \cap B$ by balls of radius $2^{-\gamma p}$ centered on $K \cap A_{p} \cap B$. Just as above, the Ahlfors regularity of $\mu$ shows that $\# I_{B} \ll 2^{\delta \gamma p} \mu\left(A_{p} \cap B\right) \ll 2^{p \gamma \delta} 2^{-p[\delta+(\gamma-1) \alpha]}$. Thus, we find for every $s>0$,

$$
\sum_{B \in \mathcal{C}_{p}} \sum_{i \in I_{B}}\left(\operatorname{diam} B_{i}\right)^{s} \ll 2^{p \delta} 2^{p(\gamma-1)(\delta-\alpha)} 2^{-p \gamma s}=2^{-p[s \gamma-\gamma \delta+\alpha(\gamma-1)]}
$$

If $s>\delta-\alpha\left(1-\frac{1}{\gamma}\right)$, then the family of balls $\left(B_{i}\right)_{i \in I_{B}, B \in \mathcal{C}_{p}, p \in \mathbb{N}}$ satisfies the assumption of the Hausdorff-Cantelli lemma, and therefore, letting

$$
s \rightarrow \delta-\alpha(1-1 / \gamma)
$$

we find that $\operatorname{dim}_{H}\left(\limsup B_{i}\right) \leq \delta-\alpha\left(1-\frac{1}{\gamma}\right)$. Now, since $\gamma<\beta$, we have $K \cap W_{\beta} \subset\left(\lim \sup B_{i}\right)$, hence letting $\gamma \rightarrow \beta$, we can conclude that the Hausdorff dimension of $K \cap W_{\beta}$ is not greater than $\delta-\alpha\left(1-\frac{1}{\beta}\right)$.

In the case of $\mathbb{Q}$-rank one, any Ahlfors-regular measure of dimension $\delta$ is $\delta$-IAD, so we get the following corollary, which applies in particular when $X=$ $\mathbb{S}^{n-1}$ is the unit sphere in $\mathbb{R}^{n}$ :

Corollary 4.9. Let $X$ be a rational quadric hypersurface of $\mathbb{Q}$-rank one, and let $\mu$ be an Ahlfors-regular measure of dimension $\delta$ on $X$. Writing $K=\operatorname{Supp} \mu$, we have, for every $\beta \geq 1, \operatorname{dim}_{H}\left(K \cap W_{\beta}\right) \leq \frac{\delta}{\beta}$.

\subsection{Badly approximable points}

Recall the definition (2.6) of the set $\mathrm{BA}_{X}$ of intrinsically badly approximable points in $X$. As was mentioned in Section 2, it is known [14] to satisfy some winning properties in the sense of Schmidt's games. Our goal will now be to give a more elementary proof of a refinement of the winning property, again using the simplex lemma. 
In order to study the properties of badly approximable numbers, Schmidt introduced in 24] a certain family of games, and the associated winning property. Those games were subsequently studied in numerous papers, among which [5] is the most relevant for the present purposes.

We now explain the principles of our version of Schmidt's game. As before, $X$ is a rational quadric hypersurface of $\mathbb{P}^{n}(\mathbb{R})$. There are two players, Alice and Bob, and some parameter $\beta \in\left(0, \frac{1}{3}\right)$. To start, Bob chooses a ball $B_{0}=$ $B\left(x_{0}, \rho_{0}\right)$ in $X$. Then, at each stage of the game, after Bob has chosen a ball $B_{i}=B\left(x_{i}, \rho_{i}\right)$, Alice chooses a totally isotropic rational subspace $L$ of $X$ and deletes its neighborhood of size $\varepsilon$, with $0<\varepsilon \leq \beta \rho_{i}$.

A set $S$ is isotropically $\beta$-winning if Alice can make sure that

$$
\bigcap B_{i} \cap S \neq \varnothing \text {. }
$$

Finally, $S$ is isotropically winning if it is isotropically $\beta$-winning for arbitrarily small $\beta>0$. This is a strengthening of the hyperplane absolute winning property as defined in [5] (for the latter, Alice is allowed to delete neighborhoods of arbitrary hyperplanes). Thus the following theorem is a refinement of [14, Theorem 4.3]:

Theorem 4.10 (Badly approximable points on $X$ are winning). Let $X$ be a rational quadric hypersurface in $\mathbb{P}^{n}(\mathbb{R})$. Then the set $\mathrm{BA}_{X}$ is isotropically winning.

Proof. Fix $\beta \in\left(0, \frac{1}{3}\right)$. Bob first picks a ball $B_{0}=B\left(x_{0}, \rho_{0}\right)$. By Lemma 3.1, there exists a constant $c>0$ depending only on $X$ such that all rational points $v$ in $2 B_{0}$ satisfying $H(v) \leq c \rho_{0}^{-1}$ are included in some totally isotropic rational subspace $L_{0}$. Alice deletes $L_{0}^{\left(\beta \rho_{0}\right)}$. Similarly, once Bob has chosen a ball $B_{i}=B\left(x_{i}, \rho_{i}\right)$, the rational points $v \in 2 B_{i}$ such that $H(v) \leq c \rho_{i}^{-1}$ all lie on a hyperplane $L_{i}$, and Alice deletes $L_{i}^{\left(\beta \rho_{i}\right)}$. If there is no rational point of small height in $B_{i}$, then Alice can delete a ball of radius $\beta \rho_{i}$ around the center. This ensures that $\rho_{i} \rightarrow 0$.

We claim that this strategy forces $\bigcap_{i \geq 0} B_{i} \subset \mathrm{BA}_{X}$. To see this, let $x \in \bigcap B_{i}$ and $v \in X(\mathbb{Q})$. Choose $i$ such that

$$
c \rho_{i-1}^{-1} \leq H(v) \leq c \rho_{i}^{-1} .
$$

If $v \notin 2 B_{i}$, then, using $x \in B_{i}$, we find

$$
\operatorname{dist}(x, v) \geq \rho_{i} \geq \beta \rho_{i-1} \geq \beta c H(v)^{-1} .
$$

And if $v \in 2 B_{i}$, then (4.3) implies that $v \in L_{i}$, and since $x \in B_{i+1}$,

$$
\operatorname{dist}(x, v) \geq \beta \rho_{i} \geq \beta^{2} \rho_{i-1} \geq \beta^{2} c H(v)^{-1} .
$$

Taking $c_{0}=c \beta^{2}$, we find

$$
\forall v \in X(\mathbb{Q}), \operatorname{dist}(x, v) \geq c_{0} H(v)^{-1},
$$

so $x \in \mathrm{BA}_{X}$. 
As is the case with the hyperplane absolute game, the advantage of the isotropic game is the inheritance of winning properties to sufficiently regular subsets. More precisely, given a compact subset $K \subset X$, we may consider the isotropic game played on $K$. The rules are the same as before, but the ambient metric space is now $K$ : at each stage, Bob chooses a ball $B\left(x_{i}, \rho_{i}\right)$ centered on $K$, and Alice deletes the intersection of $K$ with the neighborhood of size $\beta \rho_{i}$ of a rational isotropic subspace. Naturally, we shall say that a set $S$ is isotropically winning on $K$ if $S \cap K$ is winning for the isotropic game on $K$.

Following Broderick-Fishman-Kleinbock-Reich-Weiss [5], let us say that a subset $K \subset X$ is isotropically diffuse if there exists $\beta, \rho_{K}>0$ such that for every $\rho \in\left(0, \rho_{K}\right), x \in K$, and every totally isotropic subspace $L$, the set

$$
K \cap B(x, \rho) \backslash L^{(\beta \rho)}
$$

is non-empty. This is a quantitative way to say that $K$ is nowhere included in a small neighborhood of a totally isotropic subspace. The next lemma is a straightforward analogue of [5, Proposition 4.9]:

Lemma 4.11. Let $X$ be a rational quadric hypersurface in $\mathbb{P}^{n}(X)$. If $L \subset K$ are two isotropically diffuse subsets of $X$, and $S \subset X$ is isotropically winning on $K$, then $S$ is isotropically winning on $L$.

The proof is identical to the argument presented in [5], with obvious modifications to our setting; we refer the reader to [5. Section 4] for details.

It follows from the above lemma and Theorem 4.10 that $\mathrm{BA}_{X}$ is isotropically winning on any isotropically diffuse subset of $X$. This in particular applies to smooth submanifolds $M$ of $X$ of dimension not less than $\operatorname{rk}_{\mathbb{Q}}(X)$, which are clearly isotropically diffuse. Furthermore, the Lebesgue measure on $M$ as above is clearly Ahlfors-regular of dimension equal to $\operatorname{dim} M$. Therefore, in view of 5 , Lemma 5.3], for every open subset $U$ of $X$ such that $U \cap M \neq \varnothing$, one has

$$
\operatorname{dim}_{H}\left(M \cap \mathrm{BA}_{X} \cap U\right)=\operatorname{dim} M,
$$

which implies Theorem 2.5 .

Remark 4.12. In the case of $X=\mathbb{S}^{n-1}$, or more generally of a rational quadric of $\mathbb{Q}$-rank one, the above corollary shows that $\mathrm{BA}_{X}$ is winning on any positivedimensional submanifold of $X$. This can be compared with a similar question for Diophantine approximation in Euclidean spaces, for which it is still open, despite recent progress due to Beresnevich [1, and Yang [27.

\section{$5 \quad$ Further directions and open problems}

Khintchine's theorem. It would be interesting to use the geometric observations of this note to give an elementary proof of Khintchine's theorem on quadric hypersurfaces, due to Fishman, Kleinbock, Merrill and Simmons [13, Theorem 6.3].

Singular points. Given a rational quadric $X$ in $\mathbb{P}^{n}(X)$, one may define, for $c>0$,

$$
D(c)=\left\{\begin{array}{l|l}
x \in X & \begin{array}{l}
\exists N_{0}: \forall N \geq N_{0} \exists v \in X(\mathbb{Q}) \text { such that } \\
H(v) \leq N \text { and } \operatorname{dist}(x, v) \leq \frac{c}{\sqrt{N H(v)}}
\end{array}
\end{array}\right.
$$


and call a point $x \in X$ singular if $x \in \bigcap_{c>0} D(c)$. If $X$ has $\mathbb{Q}$-rank 1 , it follows from Dani's work 9 that $x$ is singular if and only if $x \in X(\mathbb{Q})$. In fact, one can show that if $X$ has $\mathbb{Q}$-rank $1, D(c)=X(\mathbb{Q})$ for $c>0$ small enough. This follows for example from the following strengthening of Lemma 3.1, whose proof is identical up to some minor changes. See also [21, Theorem 3] for an alternative proof.

Lemma 5.1 (A stronger simplex lemma for quadric hypersurfaces). Let $X$ be a rational quadric hypersurface in $\mathbb{P}^{n}(\mathbb{R})$. Then there exists $c>0$ such that, for every $x \in X$ and any $\rho \in(0,1)$, the set

$$
\left\{v \in X(\mathbb{Q}) \mid H(v) \leq c \rho^{-1}, \operatorname{dist}(x, v) \leq \sqrt{\frac{\rho}{H(v)}}\right\}
$$

is contained in a totally isotropic rational subspace $L \subset X$.

When the quadric $X$ has $\mathbb{Q}$-rank at least 2, it is natural to expect that there exist some nontrivial singular points. It might then be interesting to compute the Hausdorff dimension of the set of singular points on $X$, similarly to what has been done in 27, 8, for Diophantine approximation in the Euclidean space.

Extremality. In view of the definitive results in the area of Diophantine approximation on manifolds and fractals obtained in [18, 19, it is natural to attempt to weaken the condition of isotropic absolute decay of $\mu$ as in Theorem 4.2 . and conjecture that on a general quadric hypersurface, any analytic submanifold that is not included in an isotropic subspace is extremal. In fact, by analogy with [16, 17], one can guess that an analytic submanifold on a quadric hypersurface inherits its Diophantine exponent from the smallest totally isotropic subspace containing it. The proof of these two facts requires an appropriate quantitative non-divergence statement in the space of lattices, and should appear elsewhere.

Other projective varieties. One may wonder how general is the approach presented here, and whether it can be used to study intrinsic Diophantine approximation on varieties that are not quadric hypersurfaces. Some partial answers are given in 4, where Diophantine approximation on a generalized flag variety is studied. However, many questions remain open in this generality, for which we refer the reader to [4].

\section{References}

[1] V. Beresnevich, Badly approximable points on manifolds, Invent. Math. 202, no. 3 (2015), 1199-1240.

[2] V. Beresnevich, A. Ghosh, D. Simmons and S. Velani, Diophantine approximation in Kleinian groups: singular, extremal and bad limit points (2016), preprint available at arXiv:1610.05964

[3] V. Bernik and M. Dodson, Metric Diophantine approximation on manifolds, Cambridge Tracts in Mathematics, vol. 137, Cambridge University Press, Cambridge, 1999. 
[4] E. Breuillard and N. de Saxcé, A subspace theorem for manifolds, in preparation.

[5] R. Broderick, L. Fishman, D. Kleinbock, A. Reich and B. Weiss, The set of badly approximable vectors is strongly $C^{1}$ incompressible, Math. Proc. Cambridge Philos. Soc. 153, no. 2 (2012), 319-339.

[6] J. W. S. Cassels, An introduction to Diophantine approximation, Cambridge Tracts in Mathematics and Mathematical Physics, vol. 145, Cambridge University Press, New York, 1957.

[7] Y. Cheung, Hausdorff dimension of the set of singular pairs, Ann. Math. 173, no. 1 (2011), 127-167.

[8] Y. Cheung and N. Chevallier, Hausdorff dimension of singular vectors, Duke Math. J. 165, no. 12 (2016), 2273-2329.

[9] S. G. Dani, Divergent trajectories of flows on homogeneous spaces and Diophantine approximation, J. Reine Angew. Math. 359 (1985), 55-89.

[10] H. Davenport, A note on Diophantine approximation. II, Mathematika 11 (1964), 50-58.

[11] H. Dickinson and M. M. Dodson, Simultaneous Diophantine approximation on the circle and Hausdorff dimension, Math. Proc. Cambridge Philos. Soc. 130, no. 3 (2001), 515-522.

[12] C. Drutu, Diophantine approximation on rational quadrics, Math. Ann. 333, no. 2 (2005), 405-469.

[13] L. Fishman, D. Kleinbock, K. Merrill anf D. Simmons, Intrinsic Diophantine approximation on quadric hypersurfaces (2014), preprint available at arXiv: 1405.7650

[14] L. Fishman, D. Kleinbock, K. Merrill anf D. Simmons, Intrinsic Diophantine approximation on manifolds: general theory, Trans. Amer. Math. Soc. 370. no. 1 (2018), 577-599.

[15] L. Fishman, K. Merrill and D. Simmons, Hausdorff dimensions of very well intrinsically approximable subsets of quadratic hypersurfaces (2015), preprint available at arXiv:1502.07648v2.

[16] D. Kleinbock, Extremal subspaces and their submanifolds, Geom. Funct. Anal. 13, no. 2 (2003), 437-466.

[17] D. Kleinbock, An extension of quantitative nondivergence and applications to Diophantine exponents, Trans. Amer. Math. Soc. 360 (2008), 6497-6523.

[18] D. Kleinbock, E. Lindenstrauss and B. Weiss, On fractal measures and Diophantine approximation, Selecta Math. (N.S.) 10, no. 4 (2004), 479 523.

[19] D. Kleinbock and G. A. Margulis, Flows on homogeneous spaces and Diophantine approximation on manifolds, Ann. Math. 148, no. 1 (1998), 339-360. 
[20] D. Kleinbock and K. Merrill, Rational approximation on spheres, Isr. J. Math. 209 (2015), no. 1, 293-322.

[21] D. Kleinbock and N. Moshchevitin, Simultaneous Diophantine approximation: sums of squares and homogeneous polynomials, preprint available at arXiv:1803.09306v2, Acta Arithmetica, to appear.

[22] S. Kristensen, R. Thorn and S. Velani, Diophantine approximation and badly approximable sets, Adv. Math. 203, no. 1 (2006), 132-169.

[23] A. Pollington and S. Velani, Metric diophantine approximation and absolutely friendly measures, Selecta Math. (N.S.), 11, no. 2 (2005), 297-307.

[24] W. M. Schmidt, On badly approximable numbers and certain games, Trans. Amer. Math. Soc. 123 (1966), 178-199.

[25] W. M. Schmidt, Diophantine approximation, Lecture Notes in Mathematics, vol. 785, Springer, Berlin, 1980.

[26] B. Weiss, Almost no points on a Cantor set are very well approximable, Proc. R. Soc. Lond. A 457 (2001), 949-952.

[27] L. Yang, Badly approximable points on curves and unipotent orbits in homogeneous spaces (2017), preprint available at arXiv:1703.03461v1.

Dmitry Kleinbock,

Department of Mathematics, Brandeis University, Waltham MA 02454, USA

E-mail address: kleinboc@brandeis.edu

Nicolas de Saxcé,

CNRS, Université Paris 13 - LAGA, 93430 Villetaneuse, FRANCE

E-mail address: desaxce@math.univ-paris13.fr 